BRIEF REPORT

\title{
Estimating the incidence of hospitalized injurious falls: impact of varying case definitions
}

\section{$S$ Boufous, C Finch}

Injury Prevention 2005;11:334-336. doi: 10.1136/ip.2005.009837

Aim: To assess the validity of widely used approaches to estimate the incidence of hospitalized falls.

Methods: Internal probabilistic data linkage of the 2000-01 New South Wales Inpatient Statistics Collection was used to identify first admissions for injurious falls.

Results: Using data linkage techniques, a total of 20883 (93.9\%, 95\% Cl 93.5 to 94.2 ) cases were identified as first admission for injurious falls corresponding to an incidence rate of 1161.4 per 100000 . The exclusion of non-acute admissions approach provided the best estimate of incidence (1185.4 per 100000 people). When comparing the performance of different approaches to identifying first admissions to that of the data linkage "gold standard", the method based on the transfer variable performed best in terms of sensitivity and specificity.

Conclusions: All the examined approaches have relatively low specificity raising questions about their use. The introduction of a unique patient identifier and the date of injury in hospital discharge datasets would facilitate the identification of incident cases of fall related hospitalizations.

nternationally, hospital discharge data are used widely to determine the incidence of hospitalization for injurious falls. However, a major limitation of this is that patients who have been readmitted or transferred from one hospital to another, and in some instances even within the same hospital, for treatment of the same injury may by recorded more than once. Hospital discharge datasets usually lack unique personal identifiers to identify individual patients and information on the date of injury that would facilitate identification of multiple admissions for the same injury.

In the absence of personal identification and date of injury information, some studies have simply assumed that each discharge refers to a single episode of injury, resulting in an overestimation of the true hospitalized falls incidence rate. ${ }^{12}$ Others have used various case definitions based on variables readily available in most hospital discharge datasets, such as "readmission within 28 days", "transfer", "single day admission", and "admission to non-acute hospitals", to identify incident cases..$^{3-8}$ The aim of this study was to assess the validity and estimate the effects of these case definitions on the calculation of hospitalized falls incidence rates-that is, single admissions, as well as (in the case of multiple admissions) the first hospital admission for the same injury.

\section{METHODS}

Information on hospitalized injurious falls cases was obtained from the 2000-01 Inpatient Statistics Collection which covers all inpatient separations/discharges from public hospitals in New South Wales (NSW). Hospitals in NSW are required to submit details for every inpatient episode of care.
An episode of care ends by discharge, transfer, death, or by the patient becoming a different type of patient within the same hospital. ${ }^{9}$ Cases selected for this study included all public hospital admissions in NSW of patients aged 50 years with an ICD 10 external cause/mechanism of injury indicating a fall (W00-W10) and a primary diagnosis of injury (S00T98). ${ }^{10}$

An internal probabilistic data linkage method was adopted, using the record linkage package LinkageWiz, ${ }^{11}$ to identify multiple separations for injurious falls for each patient. The strength of the probabilistic linkage procedure is that it takes into account spelling errors and missing values in each field used for matching. ${ }^{12}$ Matching variables used in the process included the phonetic encoding of family names, middle name and given name as well as the date of birth (day, month, year), sex, address of residence (street number, street name, postcode), country of birth, language spoken at home, as well as the principal diagnosis and the external cause of injury.

The rate of first admission was examined by age, sex, and injury type. Using the first admissions identified by the record linkage as the "gold standard", we also examined the sensitivity and specificity of other classification approaches routinely used to estimate the number of first admissions of hospitalized falls. These include the exclusion of transfers, readmissions within 28 days, admissions to non-acute hospitals, and "day only" admissions.

The incidence of injurious falls leading to hospitalisation was computed for each approach using the number of NSW residents aged 50 years and over. Ninety five percent confidence intervals were computed for various proportions. All analyses were carried out using SAS (version 8.1). ${ }^{13}$

\section{RESULTS}

There were 22250 separations for injurious falls in people aged 50 years and older in NSW between the 1 July 2000 and 30 June 2001. Of these cases, 20883 (93.9\%, 95\% CI 93.5 to 94.2) cases were identified, using data linkage, as incident cases for hospitalized injurious falls.

The proportion of incident cases of fall related injuries leading to hospitalization did not vary significantly by age and sex. Similarly, although vertebral fractures, fractures of other parts of the femur, and fractures of skull and facial bones had the lowest proportions of incident cases compared with other fracture types, the difference was not statistically significant.

When comparing the performance of different approaches to identifying incident cases, compared to the data linkage, the "transfer from" variable performed best in terms of sensitivity and specificity (table 1). A combination of both "readmission within 28 days" and "transfer from" improved the specificity but reduced the sensitivity. A case definition based on the exclusion of admissions to non-acute hospitals had a relatively high sensitivity but a low sensitivity and the reverse was true for day only admissions. 
Table 1 Specificity and sensitivity of variables used to estimate incident cases of hospitalized injurious falls, compared with those identified through record linkage

\begin{tabular}{|c|c|c|c|c|}
\hline Case definition & Sensitivity $(95 \% \mathrm{Cl})$ & Specificity $(95 \% \mathrm{CI})$ & Incidence rate* $(95 \% \mathrm{Cl})$ & Change in incidence $†$ \\
\hline Exclusion of readmissions within 28 day & $91.6(91.2$ to 92.0$)$ & 55.8 (53.2 to 58.4$)$ & 1097.5 (1082.3 to 1112.8$)$ & $\downarrow 5.6 \%$ \\
\hline Exclusion of transfers & 93.9 (93.6 to 94.2$)$ & 53.0 (43.9 to 49.2$)$ & 1126.2 (1110.8 to 1141.7$)$ & $\downarrow 3.1 \%$ \\
\hline Exclusion of both readmissions and transfers & $88.6(88.2$ to 89.1$)$ & $68.3(65.8$ to 70.7$)$ & 1053.6 (1038.7 to 1068.5$)$ & $\downarrow 9.4 \%$ \\
\hline Inclusion of acute admissions only & $96.4(96.1$ to 96.6$)$ & $12.8(85.4$ to 89.0$)$ & 1185.4 (1169.6 to 1201.2$)$ & $\uparrow 2.1 \%$ \\
\hline Exclusion of day only admissions & $18.6(18.1$ to 19.1$)$ & $90.9(89.4$ to 92.5$)$ & 219.5 (212.7 to 226.4$)$ & $\downarrow 82.2 \%$ \\
\hline Internal data linkage & $100 \%$ & $100 \%$ & $1161.4(1145.8$ to 1177.1$)$ & - \\
\hline All fall related separations & - & - & 1237.5 (1221.3 to 1253.6$)$ & $\uparrow 6.6 \%$ \\
\hline
\end{tabular}

*Incidence rate per 100000 people aged 50 years and over.

†Compared with the incidence obtained based on data record linkage.

When examining the impact of these approaches on the incidence of hospitalized injurious falls, the exclusion of nonacute admissions approach provided the best estimate of incidence compared to that provided by data linkage (table 1).

\section{DISCUSSION}

This is the first study to examine the validity of various approaches to identifying first hospital admissions for injurious falls. Using data linkage methods, $93.9 \%$ of hospital separations for injurious falls were found to correspond to first admissions/incident cases. A similar proportion was found in two other studies using different methods. ${ }^{14}{ }^{15}$

In the absence of more specific variables such as a unique personal identifier and the date of injury, our results suggest that case identification approaches based on readmission/ transfer variables, particularly when used in combination, may provide the most accurate identification of incident falls leading to hospitalization. However, the incidence rate of hospitalized injurious falls when using this approach was 9.4\% lower than that based on data record linkage, raising questions about its utility in estimating incident cases.

According to coding guidelines, the "readmission within 28 days" variable should be used to indicate a readmission for the same problem/condition. ${ }^{16}$ However, it is possible that some hospitals/coders may be using this even when a previous admission is related to other injuries or health conditions. The identification method based on "day only admissions" had a very low sensitivity and is simply not suitable for estimating incident cases of hospitalized injurious falls. This is reflected in the lower incidence rate found when using this method compared to the "gold standard".

Although the inclusion of "acute admissions only" had a low specificity, it yielded a high sensitivity and a comparable incidence rate (only $2 \%$ higher) to that resulting from the use of probabilistic data linkage. Although sensitivity is important in this context, as it is more indicative of "true" first admissions/incident cases, it is equally important to achieve a high specificity and avoid false positives, as various attributes might change between first and subsequent admissions.

The unsatisfactory validity (specificity in particular), in relation to data record linkage, of the variables readily available in most hospital datasets indicates that alternative methods to identify incident cases of injurious falls admitted to hospitals are needed. In the state of California, only first admissions for an injury are assigned an external cause which means that by selecting records with an external injury code, only incident cases are selected and multiple counting of cases is avoided. ${ }^{17}$ However, this method is limited when the aim is to measure hospital bed use and the economic impact of injury on the delivery of health care.

As probabilistic record linkage is far from being practical in identifying repeat admissions, the use of a unique patient identifier coupled with the date of injury remains the most valid and accurate method of identifying incident cases of injurious falls as well as other conditions. ${ }^{14}$ Unique patient identifiers have the potential to not only identify incident cases for various hospitalized health conditions but to also ensure the safety of patient care by enabling access to patient encounter information across the continuum of care. ${ }^{18}{ }^{19}$ Routine recording of the "date of injury" would further simplify the process of determining whether these readmissions are for the same injury or for a new injury which might have occurred at a later date. This is particularly relevant to falls in older people where more than one fall can occur in a given year.

Although efforts have been made to achieve an accurate linked dataset, data record linkage involves trade-offs between the number of false positives and false negatives and the inclusion of such cases in the final dataset is inevitable. However, previous studies have shown that probabilistic record linkage techniques, similar to those used in this study, result in high quality outcomes and that the process results in no more than a $1 \%$ error rate. ${ }^{20}$ This is supported by the findings of another study, which used a unique personal identifier and date of injury, and found the same proportion of incident cases of hospitalized injurious falls as our study. ${ }^{14}$

In conclusion, all the approaches using variables readily available in most hospital discharge datasets to estimate incident cases of hospitalised injurious falls have relatively low specificity, raising questions about their use. This emphasizes the importance of the introduction of a unique patient identifier and the date of injury in providing a more accurate picture of incident cases of injury related hospitalizations, including those resulting from falls.

\section{ACKNOWLEDGEMENTS}

S Boufous was supported by the NSW Injury Risk Management Research Centre's core funding provided by the NSW Department of Health, the NSW Roads and Traffic Authority, and the Motor Accidents Authority. C Finch was supported by a National Health and Medical Research Council Principal Research Fellowship. The authors wish to thank the Centre for Epidemiology and Research at the NSW Health Department for providing the data for this study.

\section{Key points}

- Approaches based on the exclusion of non-acute cases and transfers provided the best estimate of incidence of hospitalized injurious falls.

- Compared to data linkage, all the examined approaches have relatively low specificity raising questions about their use.

- The introduction of a unique patient identifier and the date of injury would facilitate the identification of incident cases of fall related hospitalizations. 


\section{Authors' affiliations}

S Boufous, C Finch, NSW Injury Risk Management Research Centre, University of New South Wales. Sydney, Australia

Correspondence to: Mr S Boufous, the NSW Injury Risk Management Centre. Building G2, Western Campus, University of New South Wales, Sydney NSW 2052 Australia; soufiane@unsw.edu.au

Accepted 7 September 2005

\section{REFERENCES}

1 Wigg AE, Hearn TC, McCaul KA, et al. Number, incidence, and projections of distal forearm fractures admitted to hospital in Australia. J Trauma 2003;55:87-93.

2 Schmertmann ML, Williamson A. NSW injury profile: A review of injury data (1995-1999) and identification of areas requiring further study. Sydney: NSW Injury Risk Management Research Centre, 2002.

3 Jacobsen SJ, Goldberg J, Miles TP, et al. Hip fracture incidence among the old and very old: a population-based study of 745,435 cases. Am J Public Health 1990;80:871-3

4 Lau EM, Cooper $\mathrm{C}$, Fung $\mathrm{H}$, et al. Hip fracture in Hong Kong over the last decade-a comparison with the UK. J Public Health Med 1999;21:249-50.

5 Watt GM, Ozanne-Smith J. Trends in public hospital injury admission rates Victoria, July 1986-1991. Aust N Z J Public Health 1996;160:790-4.

6 Cripps R, Carman J. Falls by the elderly in Australia: trends and data for 1998. Injury Research and Statistics Series. Adelaide: Australian Institute of Health and Welfare, 2001.

7 Boufous S, Finch C, Lord S. The incidence of hip fracture in NSW: are our efforts having an effect? Med J Aust 2004;180:623-6.
8 Lord S. Hip fractures: Changing patterns in hospital bed use in NSW between 1979 and 1990. Aust N Z J Surg 1993;63:352-5.

9 Epidemiology and Surveillance Branch. Health Outcomes and Information Statistical Toolkit (HOIST). Inpatient Statistics Collection. Sydney: NSW Department of Health, 2004.

10 National Centre for Classification in Health. The International Statistical Classification of Diseases and Related Health Problems, 10th Revision, Australian Modification (ICD-10-AM). Second edition. Sydney: National Centre for Classification in Health, 2000.

11 LinkageWiz. Record linkage software. Version 4.0.23. Adelaide: LinkageWiz Inc, 2004.

12 Clark DE. Practical introduction to record linkage for injury research. Inj Prev 2004;10:186-91.

13 SAS Institute. SAS: statistical software. Version 8.02. Cary: SAS Institute Inc, 2000;(NC).

14 Langley J, Stephenson S, Cryer C, et al. Traps for the unwary in estimating person based injury incidence using hospital discharge data. Inj Prev 2002:8:332-7

15 Boufous S. Williamson A. Reporting of the incidence of hospitalised injuries: numerator issues, Inj Prev, 2003;9, 370-5.

16 NSW Health. ISC Instruction Manual 2001/2002. Public Sector Inpatient Facilities. Available at http://www.health.nsw.gov.au/im/ims/isc/ instructions-public-2001-2002.html (accessed December 2004).

17 Ellis AA, Trent RB. Hospitalized fall injuries and race in California. Inj Prev $2001 ; 7: 316-20$.

18 National Health Information Management Group. Issues for the use of unique patient identifiers in statistical collections. Canberra: Australian Institute of Health and Welfare, 2002.

19 Information Management Implementation Co-ordination Group. Government's Action Plan for Health. A NSW Health Strategy for the Electronic Health Record. Sydney: NSW Health, 2001.

20 Rosman DL, Knuiman MW. A comparison of hospital and police road injury data. Accid Anal Prev 1994;26:215-22.

International Society for Child and Adolescent Injury Prevention Bi-Annual Conference: 5-6 April 2006, Durban, South Africa

Injury and violence are major causes of child and adolescent death and disability throughout the world. Despite a growing body of evidence that injury is largely preventable, few evidence based interventions are implemented, particularly in the developing world. This conference will address this gap between evidence and practice by bringing together the world's leaders in pediatric and adolescent injury and violence prevention as a joint consultation of the World Health Organization (WHO) and the International Society for Child and Adolescent Injury Prevention (ISCAIP).

The conference will be co-sponsored by the United States Centers for Disease Control and Prevention.

Objectives

- Identify key issues for child injury research in low and middle income countries.

- Contribute to the WHO Global Strategy for Childhood Injury Prevention.

Program highlights

- International injury and violence experts and practitioners sharing evidence and experience on prevention strategies.

- Presentations from the WHO on the Global Strategy for Childhood Injury Prevention.

- Discussion on recommendations for implementation of prevention strategies in low and middle income countries.

- Research forum highlighting current initiatives and findings in child and adolescent injury and violence from around the world.

For more information, please contact the conference organizers, the Continuing Medical Education Department at The Children's Hospital of Philadelphia. Tel: +1215590 5263; fax: +1 215590 4342; email: utsch@email.chop.edu; website: http://www.chop.edu/cme 


\section{LETTERS}

\section{Resources for terror and road injury prevention}

We question the ethics and logic underlying the thesis that a shift of resources from terror prevention and deterrence to road injury prevention is dictated by the huge differences between the relatively small death tolls from terror and the large death tolls from road injury in OECD countries.'

The follow up period (1994-2003) is cut off before the terror attacks in Madrid (191 dead) and London (52 dead), and the tolls do not include overseas deaths of OECD citizens, as in the attacks in Bali, Egypt, and Tunisia. The boundaries exclude Israel (a member of WHO Europe and signatory to many EU agreements) where over 550 civilians were killed in terror attacks between September 2000 and January 2003. ${ }^{2}$

The study ignored the victims of the Moscow theater attack, the 300 victims of terror in Beslan, most of whom were children, and the thousands of civilian dead in Iraq from terror attacks. Genocidal terror in prosperous countries cannot be insulated from global terrorism, given the remarkable ability of its vectors-the perpetrators-to move around the global village.

In road injury prevention, the barrier to major progress in reducing death tolls is not budget, but the direction and content of injury prevention programs. Speed camera networks in Victoria, Australia and in the UK have reduced road death tolls by some $40-$ $50 \%$ in the last decade, and themselves pay for their operation. ${ }^{34}$ By contrast, prevention of terror is cost intensive, since the results have to be failsafe.

Indeed it could well be that low death rates from terror in OECD countries are precisely a result of the massive investments in terror prevention. The bizarre logic used by the authors would justify neglecting the upkeep and maintenance of the dykes in the Netherlands, because that country has had no floods in recent years. It would lead to the suspension of cost intensive failsafe airport and airplane security for aircraft flying to and from Israel because Israel has not experienced a hijacking since 1972. If there have been no more $9 / 1$ ls in the USA, it is quite possibly a result of the costly interventions which this paper questions.

E D Richter, T Berman

Hebrew University-Hadassah School of Community Medicine and Public Health, Injury Prevention Center, Jerusalem, Israel

Correspondence to: Dr E D Richter, Hebrew University-Hadassah School of Community Medicine and Public Health, Injury Prevention Center, 91120 Jerusalem, Israel; elir@cc.huji.ac.il

doi: 10.1136/ip.2006.011577

Accepted 1 February 2006
References

1 Wilson N, Thompson G. Deaths from international terrorism compared with road crash deaths in OECD countries. Inj Prev 2005; 1 1:332-3.

2 Institute for Counter-Terrorism. International Terrorism Database. Available at http:// www.ict.org.il/ (accessed February 2006).

3 PA Consulting Group. The National Safety Camera Programme: four-year evaluation report. December 2005. Available at http://www.dft. gov.uk/stellent/groups/dft_rdsafety/documents/ downloadable/dft_rdsaf ety_610816pdf.

4 Richter ED, Friedman LS, Berman T, et al. Death and injury from motor vehicle crashes a tale of two countries. Am J Prev Med 2005;29:440-9.

\section{Climate change control and injury prevention: more win-win solutions}

The journal recently published an excellent special feature on climate change and injury prevention by Roberts and Hillman. ${ }^{1}$ The authors detailed a number of "win-win solutions" whereby actions to prevent climate change were also likely to contribute to injury prevention. However, their list was incomplete and we wish to point out some additions:

- The use of carbon charges on fossil fuels would be likely to reduce injuries for two reasons. The first is the historical evidence around petrol prices and motor vehicle fatalities; ${ }^{23}$ the second is that, if the carbon charge were not offset by other tax reductions, then it would raise revenue that could be used to invest in improved public transport systems. That would help lower injury rates, because public transport is safer than private vehicle use. The Intergovernmental Panel on Climate Change has also reported that controlling road traffic would benefit health through reductions in road traffic crashes.

- Reducing domestic hot water temperatures can prevent scalding injuries ${ }^{4}$ and also save both energy and financial resources.

- The introduction (and extension) of daylight saving schemes in many countries can save both energy and potentially reduce motor vehicle and pedestrian injury rates. ${ }^{5}$

If Western economies transmitted price incentives via carbon charges, then demand reduction, increased energy efficiency, and substitution of non-fossil fuel renewables for oil would decrease Western dependence on Middle East oil. This in turn could reduce the perceived need for military interference by Western powers in the Middle East and elsewhere, and reduce the risk of deaths and injuries from both military operations and international terrorist attacks.

Collectively, these additional "win-win solutions" may have substantive impacts on preventing injuries as well as contributing to a lowering of greenhouse gas production.
N Wilson

Department of Public Health, Wellington School of Medicine \& Health Sciences, Wellington,
New Zealand

C Wallace

School of Government, Victoria University of Wellington, Wellington, New Zealand

Correspondence to: Dr N Wilson, PO Box 7343 Wellington, New Zealand; nwilson@actrix.gen.nz doi: 10.1136/ip.2006.011650

Accepted 31 January 2006

\section{References}

1 Roberts I, Hillman M. Climate change: the implications for policy on injury control and health promotion. Inj Prev 2005;11:326-9.

2 Leigh JP, Frank AL. Gas taxes and motor vehicle fatalities. J Health Polit Policy Law 1988;13:723-34.

3 Scuffham PA, Langley JD. A model of traffic crashes in New Zealand. Accid Anal Prev 2002;34:673-87.

4 Ytterstad B, Smith GS, Coggan CA. Harstad injury prevention study: prevention of burns in young children by community based intervention. Inj Prev 1998;4:176-80.

5 Ferguson SA, Preusser DF, Lund AK, et al. Daylight saving time and motor vehicle crashes: the reduction in pedestrian and vehicle occupant fatalities. Am J Public Health 1995;85:92-5.

\section{CORRECTIONS}

doi: 10.1136/ip.2006.009076.corrl

Several errors occurred in the paper by Fingerhut and Warner in the last issue of the journal (Inj Prev 2006;12:24-9). Six cells of the IMD matrix shown in Figure 1 have been changed to display the correct codes. The cells that have changed are as follows: vertebral column and internal organ injury; vertebral column and other specified injury; multiple body regions and unspecified injury; multiple injuries and upper extremity injuries; multiple injuries and other lower extremity injuries and other lower extremity and other specified injury. The correct table is on the Injury Prevention website: http://ip. bmjjournals.com/cgi/content/full/12/1/24/DC 1

doi: 10.1136/ip.2005.009837.corr 1

Several errors occurred in the paper by Boufous and Finch in the December 2005 issue of the journal (Inj Prev 2005;11:334-6). In table 1 the row entitled "Exclusion of day only admission" has been changed to display the correct figures. The correct table is on the Injury Prevention website: http://ip.bmjjournals. com/cgi/content/full/1 1/6/334/DC 1 